\title{
Potential for leadless left bundle branch pacing for cardiac resynchronization: a case report
}

\author{
Mark Elliott ${ }^{1}$, Baldeep Sidhu ${ }^{1}$, Lucy Jarrett-Smith ${ }^{2}$, Vishal Mehta ${ }^{1}$, Justin Gould ${ }^{1}$, Angela \\ Lee $^{1}$, Steven Niederer ${ }^{1}$, and Christopher Rinaldi ${ }^{2}$ \\ ${ }^{1}$ King's College London \\ ${ }^{2}$ Guy's and St Thomas' NHS Foundation Trust
}

January 7, 2021

\begin{abstract}
Introduction: Left bundle branch pacing is a recently described form of conduction system pacing which can correct left-bundle branch block and deliver cardiac resynchronization therapy (CRT). The WiSE-CRT system delivers leadless endocardial pacing and can improve symptoms and left ventricular remodelling in CRT non-responders. Case Report: We describe the case of a 57 year old male who underwent implantation of the WiSE-CRT system after failed conventional CRT. Pacing the left bundle during implant achieved superior electrical resynchronization and equivalent hemodynamic response compared to pacing the lateral wall. Conclusion: This case demonstrates the potential for leadless left bundle branch pacing.
\end{abstract}

Potential for leadless left bundle branch pacing for cardiac resynchronization: a case report Authors:

Mark K Elliott MBBS ${ }^{1,2}$, Baldeep Singh Sidhu BM ${ }^{1,2}$, Lucy Jarrett Smith MSc ${ }^{2}$, Vishal Mehta MBBS ${ }^{1,2}$, Justin Gould MBBS $\mathrm{PhD}^{1,2}$, Angela W C Lee $\mathrm{PhD}^{1}$, Steven Niederer $\mathrm{PhD}^{1}$ and Christopher A Rinaldi MD FHRS $^{1,2}$

${ }^{1}$ School of Biomedical Engineering and Imaging Sciences, King's College London, UK

${ }^{2}$ Guy's and St Thomas' NHS Foundation Trust, London, UK

Corresponding Author:

Dr Mark Elliott

School of Biomedical Engineering and Imaging Sciences

St Thomas' Hospital

London, SE1 7EH, UK

Email: mark.elliott@kcl.ac.uk

\section{Funding and Conflicts of Interest:}

The study was supported by the Wellcome/EPSRC Centre for Medical Engineering [WT203148/Z/16/Z]. BSS is supported by a project grant from NIHR and has received speaker fees from EBR systems. Outside of the submitted work MKE, VM and JG have received fellowship funding from Abbott. CAR receives research funding and/or consultation fees from Abbott, Medtronic, Boston Scientific, Spectranetics and MicroPort outside of the submitted work. The remaining authors have nothing to disclose. 


\begin{abstract}
:
Introduction:

Left bundle branch pacing is a recently described form of conduction system pacing which can correct left-bundle branch block and deliver cardiac resynchronization therapy (CRT). The WiSE-CRT system delivers leadless endocardial pacing and can improve symptoms and left ventricular remodelling in CRT non-responders.
\end{abstract}

\title{
Case Report:
}

We describe the case of a 57 year old male who underwent implantation of the WiSE-CRT system after failed conventional CRT. Pacing the left bundle during implant achieved superior electrical resynchronization and equivalent hemodynamic response compared to pacing the lateral wall.

\section{Conclusion:}

This case demonstrates the potential for leadless left bundle branch pacing.

\section{Key Words}

Cardiac resynchronization therapy, Endocardial pacing, Leadless pacemaker, Conduction system pacing, Left bundle branch pacing.

\section{Introduction:}

Left bundle branch pacing (LBBP) has been proposed as an alternative to His bundle pacing, and has been shown to correct left bundle branch block (LBBB) and deliver cardiac resynchronization therapy (CRT) in small observational studies.[1,2] The WiSE-CRT system (EBR systems, Sunnyvale, CA) provides leadless endocardial left ventricular (LV) pacing and improves symptoms and LV remodelling in CRT nonresponders. [3,4] The system was originally designed to deliver a leadless electrode to the endocardium of the LV lateral wall to achieve CRT. We describe a case of a patient undergoing leadless LV endocardial pacing following failed conventional CRT. Assessment of different LV endocardial pacing sites prior to electrode implantation revealed stimulation of the LV septum at the site of the left bundle resulted in optimal electrical and hemodynamic indices. This case demonstrates the potential for conduction system pacing by leadless stimulation of the left bundle branch.

\section{Case Report:}

A 57-year-old male with ischaemic cardiomyopathy (LV ejection fraction 32\%), prior anterior myocardial infarction, and dual-chamber pacemaker for complete heart block underwent upgrade to a CRT-defibrillator. The upgrade procedure was difficult due to small calibre coronary sinus tributaries. The LV lead was placed in a lateral branch of the coronary sinus, however during routine follow-up the lead threshold increased with eventual loss of capture. Further transvenous attempts were unlikely to be successful given the difficulty of the original procedure and therefore we proceeded with leadless endocardial pacing using the WiSE-CRT system as part of an observational cohort study (clinicaltrials.gov NCT03495505). The patient provided written consent for the study, which was approved by local ethics committee.

The WiSE-CRT system was implanted using a previously described technique with a retrograde transaortic approach.[3] Prior to implantation of the LV electrode, different myocardial locations were tested using a roving decapolar catheter (6-F Livewire $115 \mathrm{~cm}$, St Jude Medical, Inc., St Paul, Minnesota) to assess for the optimal acute hemodynamic response (AHR) and paced QRS duration. Hemodynamic assessment (LV $\mathrm{dP} / \mathrm{dt}_{\max }$ ) was performed with a PressureWire X (Abbott, CA, USA) in the LV cavity using a previously described protocol.[5] LV dP/ $\mathrm{dt}_{\max }$ measurements were recorded using CoroFlow (Coroventis, Uppsala, Sweden) and AHR was expressed as percentage improvement from baseline dual-chamber right ventricular (RV) pacing to pacing performed at different LV endocardial sites. ECGs were recorded simultaneously. LBBP was achieved by pacing the left ventricular aspect of the interventricular septum (figure 1a) at the site of a left bundle potential (figure 2). AHR for different endocardial electrode locations are shown in 
table 1. An AHR of [?]10\% is considered to be a significant response and has been shown to be predictive of LV remodelling.[5] The greatest AHR was seen when pacing the left bundle (34\% increase from baseline) and was equivalent to biventricular endocardial stimulation at the mid-lateral wall, although LBBP showed greater electrical resynchronization (QRS duration 106ms, compared to pacing at the mid-lateral LV wall (132ms) and baseline RV-pacing (172ms) as shown in figure 3 ). The endocardial electrode was deployed in the mid-lateral wall of the LV as the current WiSE-CRT system is not designed to target the left bundle (figure 1b).

\section{Discussion:}

Both conventional (epicardial) and endocardial CRT deliver two non-physiological wave-fronts which merge to resynchronize the myocardium. Conduction system pacing can recruit the intrinsic His-Purkinje system and correct LBBB. His bundle pacing is feasible in delivering CRT in heart failure patients, with electrical resynchronization and AHR superior to conventional CRT.[6] However, it may be limited by elevated pacing thresholds at follow-up. LBBP has been more recently proposed as a method to correct LBBB at lower thresholds $[1,2]$ and is usually achieved with delivery tools and techniques for His bundle pacing, with the electrode fixed deep in the interventricular septum via a right ventricular approach. The ability to perform temporary LBBP via a retrograde trans-aortic approach has been demonstrated, with favourable hemodynamics[7] however permanent placement of a lead to the LV septum is not feasible due to the risk of embolic stroke. The WiSE-CRT system does not suffer from this drawback as the device becomes fully endothelialized and therefore does not pose a long-term risk of embolism.

In this case, LBBP was associated with excellent electrical resynchronisation (QRS duration 106ms) and hemodynamic indices. Entirely leadless CRT systems are an attractive option in patients with vascular access issues, such as hemodialysis patients, and in those with recurrent lead complications. While the majority of WiSE-CRT systems are implanted in patients with standard right-sided pacing systems, there have been reports of entirely leadless systems using the WiSE-CRT system in conjunction with a leadless pacemaker in the right ventricle. [8] The current delivery system is designed for implantation of the LV electrode in the lateral wall and rotation of the delivery catheter to reach the septum is technically challenging. Therefore, if leadless LBBP is to be pursued, new delivery systems will likely need to be developed. Furthermore, the endocardial electrode has not been specifically designed to ensure that the $3.6 \mathrm{~mm}$ barb will sufficiently penetrate from the endocardial surface down to the Purkinje tissue within the septum. The left bundle branch sits closer to the LV aspect of the septum, and LBBP from a right ventricular approach requires deep penetration into the septum, with a reported range of $11-18 \mathrm{~mm}$ in an observational study of 100 patients.[2] It is therefore likely that a left ventricular approach requires more superficial penetration, but further evaluation is needed.

\section{Conclusions:}

In this case, electrical resynchronization appeared superior during LBBP, compared to endocardial pacing in the lateral LV wall, with similar hemodynamic responses. Further studies are required to assess if leadless LBBP is a feasible and effective strategy to deliver CRT.

\section{References}

1. Zhang W, Huang J, Qi Y, et al. Cardiac resynchronization therapy by left bundle branch area pacing in patients with heart failure and left bundle branch block. Heart Rhythm . 2019;16(12):1783-1790.

2. Vijayaraman P, Subzposh FA, Naperkowski A, et al. Prospective evaluation of feasibility and electrophysiologic and echocardiographic characteristics of left bundle branch area pacing. Heart Rhythm . 2019;16(12):1774-1782.

3. Reddy VY, Miller MA, Neuzil P, et al. Cardiac Resynchronization Therapy With Wireless Left Ventricular Endocardial Pacing: The SELECT-LV Study. J Am Coll Cardiol . 2017;69(17):2119-2129.

4. Sieniewicz BJ, Betts TR, James S, et al. Real-world experience of leadless left ventricular endocardial car- 
diac resynchronization therapy: A multicenter international registry of the WiSE-CRT pacing system.Heart Rhythm . 2020;17(8):1291-1297

5. Duckett SG, Ginks M, Shetty AK, et al. Invasive acute hemodynamic response to guide left ventricular lead implantation predicts chronic remodeling in patients undergoing cardiac resynchronization therapy. $J$ Am Coll Cardiol . 2011;58(11):1128-1136.

6. Arnold AD, Shun-Shin MJ, Keene D, et al. His Resynchronization Versus Biventricular Pacing in Patients With Heart Failure and Left Bundle Branch Block. J Am Coll Cardiol . 2018;72(24):3112-3122.

7. Salden FCWM, Luermans JGL., Westra SW, et al. Short-Term Hemodynamic and Electrophysiological Effects of Cardiac Resynchronization by Left Ventricular Septal Pacing. J Am Coll Cardiol. 2020;75(4):347359

8. Sidhu BS, Gould J, Porter B, et al. Completely Leadless Cardiac Resynchronization Defibrillator System. JACC Clin Electrophysiol . 2020;6(5):5-6.

Figure 1. A: Catheter positions during temporary left bundle branch pacing. B: Final endocardial electrode placement in mid-lateral wall of the LV. The existing CS lead is noted to be very proximal in position, with the proximal pole likely located in the main body of the coronary sinus, which explains the high lead capture threshold and eventual failure.

CRT: cardiac resynchronization therapy; CS: coronary sinus; ICD: implantable cardioverter defibrillator; LV: left ventricular; RAO: right anterior oblique; RA: right atrial; RV: right ventricular.

Figure 2. Surface ECG and intracardiac electrograms from the roving decapolar catheter during baseline dual-chamber right ventricular pacing. The decapolar catheter is positioned on the left ventricular aspect of the septum and dipoles are ordered from distal (1-2) to proximal (9-10). The retrograde left bundle potential is marked with arrows on poles 1-2. Sweep speed $100 \mathrm{~mm} / \mathrm{s}$.

Table 1. Acute hemodynamic response with biventricular pacing for different left ventricular pacing locations. Acute hemodynamic response is expressed as a percentage increase in $\mathrm{dP} / \mathrm{dt}_{\max }$ compared to baseline right ventricular pacing.

Figure 3. Surface ECGs. A: Right ventricular pacing. B: Biventricular pacing with electrode at mid-lateral wall of left ventricle. C: Left bundle branch pacing.

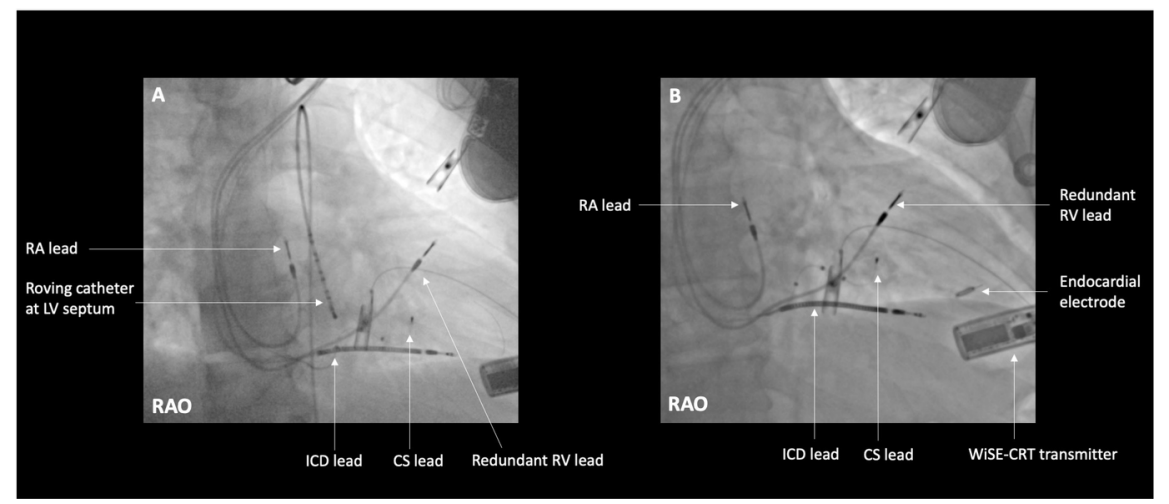




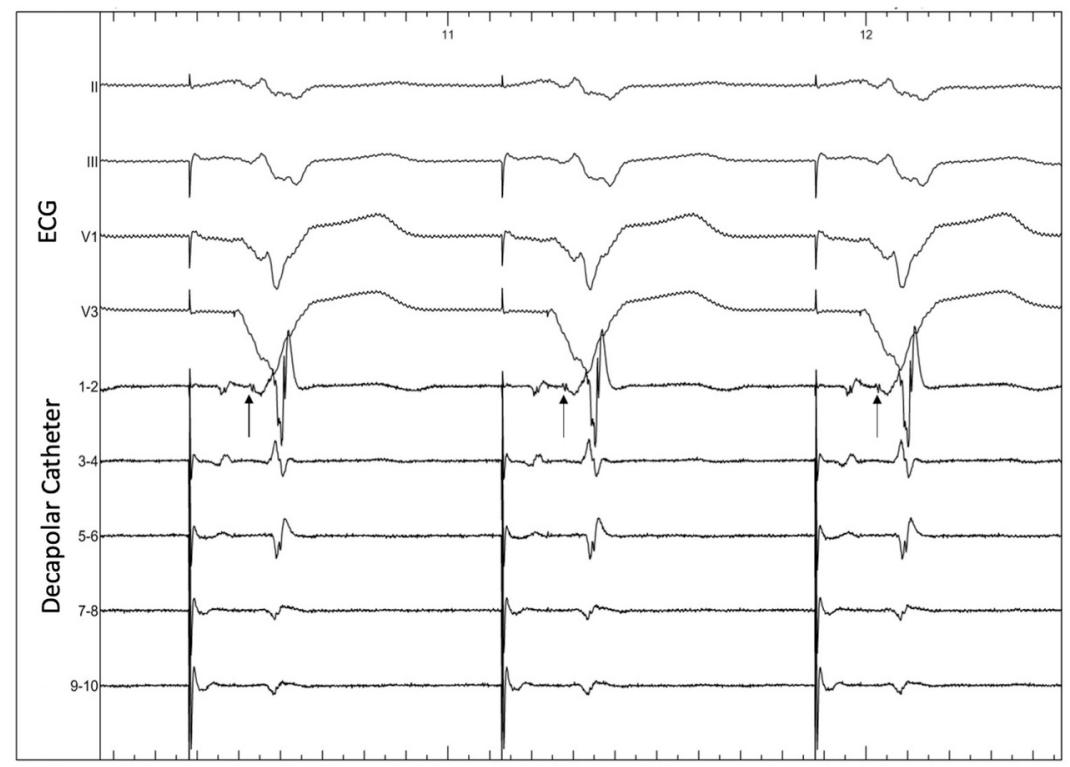

\begin{tabular}{|c|c|}
\hline Pacing Location in Left Ventricle & Acute Hemodynamic Response (\%) \\
\hline Basal lateral & 27 \\
\hline Mid lateral & 34 \\
\hline Apical septum & 22 \\
\hline Left bundle & 34 \\
\hline
\end{tabular}




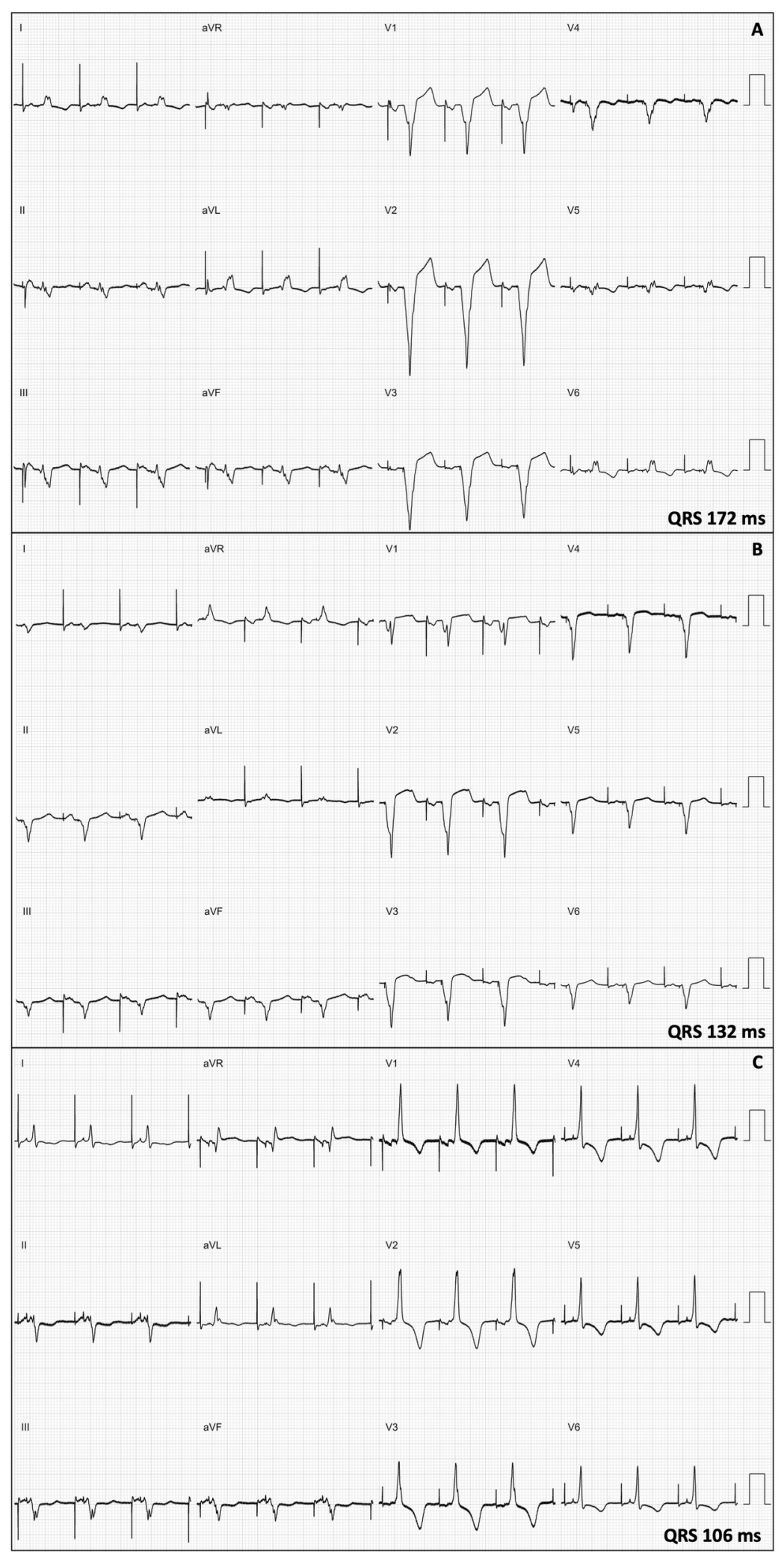

